\title{
Bipolar Spectrum Disorder May Be Associated With Family History of Diseases
}

\author{
Sermin $\underline{\text { Kesebir }}^{\mathrm{a}, \mathrm{c}}$, Merve Iris $\underline{\operatorname{Koc}}^{\mathrm{b}}$, Ahmet $\underline{\text { Yosmaoglu }}^{\mathrm{b}}$
}

\begin{abstract}
Background: This study aims at investigating into the presence of family history of diabetes, ischemic heart disease, thyroid disease, cancer, cerebrovascular disease, and epilepsy in bipolar patients.

Methods: Totally 1,148 patients admitted to our outpatient unit between January 2018 and January 2020, who were diagnosed with bipolar disorder according to Diagnostic and Statistical Manual of Mental Disorders, fifth edition (DSM-V), from whom informed consent was obtained, were cross-sectionally and consecutively evaluated. Each patient was questioned regarding a family history of diabetes, ischemic heart disease, thyroid disease, cancer (gastrointestinal, breast and prostate cancer, leukemia, and lymphoma), cerebrovascular disease and epilepsy in first- and second-degree relatives.
\end{abstract}

Results: Diabetes, ischemic heart disease, cancer, cerebrovascular disease and epilepsy were more common in the family histories than in bipolar patients. A strong correlation was found between family history positive for epilepsy and bipolar disorder with psychotic symptoms. Also, a correlation was found between family history for diabetes and seasonal course and family history positive for thyroid disease and comorbid anxiety disorder.

Conclusions: This study is the first to investigate into the frequency of physical diseases in the family histories of bipolar patients. Current therapies target the association between common leading pathways and symptoms whereas it is the association between stress and neural circuits that underlie the pathophysiology that should be targeted.

Keywords: Bipolar disorder; Family history; Inflammation; Autoimmunity; Diabetes; Vascular disease; Cancer; Epilepsy

Manuscript submitted March 21, 2020, accepted April 1, 2020

aDepartment of Psychiatry, Uskudar University, NPIstanbul Brain Hospital, Istanbul, Turkey

bUskudar University, NPIstanbul Brain Hospital, Istanbul, Turkey

${ }^{\mathrm{c} C o r r e s p o n d i n g ~ A u t h o r: ~ S e r m i n ~ K e s e b i r, ~ D e p a r t m e n t ~ o f ~ P s y c h i a t r y, ~ N P I s t a n-~}$ bul Brain Hospital, Ahmet Tevfik Ileri C. N: 18, 34768 Umraniye, Istanbul, Turkey. Email: serminkesebir@hotmail.com

doi: https://doi.org/10.14740/jocmr4143

\section{Introduction}

Current classification systems evaluate psychiatric disorders cross-sectionally and ignore the longitudinal course of disorders and the family histories of patients. In this approach, temperamental features and neurobiological heterogeneity are ignored $[1,2]$. Mood disorders can be thought of as a continuum [3]. If family history is included, a spectrum model involving medical illness is possible. In the post-insemination embryonic period yolk sac-derived erythro-myeloid progenitors enter the central nervous system (CNS) to form microglia. Microglia are immune system cells in the glial tissue surrounding the neurons. The function of microglia is to preserve and support the neuron and synapse, and in the long run, to contribute maintenance of a necessary homeostasis. A somatic mutation in BRAF gene of these stem cells disrupts the continuity of macrophage activation in adult life [4]. This change is well tolerated by many tissues without resulting in leukemia or tumor. However, it may cause relatively late onset, progressive, highly heritable neurodegenerative diseases in the brain and cerebellum, which can be prevented by BRAF inhibitors. Colony-stimulating factor-1 (CSF-1) inhibitors which are also used in cancer immunotherapy have been demonstrated in the laboratory settings to improve pathology in neurodegenerative disease models such as Alzheimer's disease.

On the other hand, we know that acute and chronic leukemias, breast, prostate, pancreas and other cancers of the digestive system such as carcinoid and proliferative tumors are more common in people with bipolar affective disorder than in healthy individuals [5]. According to epigenetic principles, Carney syndrome, multiple endocrine neoplasia (type I and II), breast and prostate cancer, carcinoid tumors and vascular diseases are found to be associated with mood disorders [6]. A comorbidity rate of more than $50 \%$ was found between these conditions and mood disorders, and a correlation of around 0.8 between these conditions and the duration of disorder was revealed [7]. There are intersections between autoimmune disorders, allergic disorders and these conditions. Epilepsy is another physical condition associated with mood disorders [8].

In 1879, in Pathology of the Mind, Maudsley stated: "Diabetes is a disease which often shows itself in families in which insanity prevail". This study aims at investigating the family history of diabetes, ischemic heart disease, thyroid disease and cancer (gastrointestinal cancers, breast and prostate cancer, 
Table 1. Rate of Medical Disease in Bipolar Patients and Their Relatives

\begin{tabular}{llll} 
& Bipolar patients (n, \%) & Relatives (n, \%) & P value \\
\hline Diabetes & $324,28.2 \%$ & $728,63.4 \%$ & 0.001 \\
Ischemic heart disease & $104,9 \%$ & $704,61.3 \%$ & 0.001 \\
Thyroid disorder & $264,22.9 \%$ & $172,14.9 \%$ & 0.042 \\
Breast, prostate, GI cancers & $80,7.2 \%$ & $532,46.3 \%$ & 0.001 \\
Leukemia and lymphoma & $20,1.5 \%$ & $84,7.3 \%$ & 0.050 \\
Cerebrovascular disease & $64,5.6 \%$ & $336,29.2 \%$ & 0.005 \\
Epilepsy & $44,3.8 \%$ & $124,10.8 \%$ & 0.053 \\
\hline
\end{tabular}

GI: gastrointestinal.

leukemia, and lymphoma), cerebrovascular disease, and epilepsy in patients with bipolar affective disorder.

\section{Materials and Methods}

In this study, 1,148 patients admitted to our outpatient unit between January 2018 and January 2020, diagnosed with bipolar affective disorder according to the Diagnostic and Statistical Manual of Mental Disorders, fifth edition (DSM-V) criteria, from whom informed consent was obtained, were cross-sectionally and consecutively evaluated.

Medical histories of the patients were taken in order. Each patient is questioned regarding a family history of diabetes, ischemic heart disease, thyroid disease, cancer (gastrointestinal cancers, breast and prostate cancer, leukemia, and lymphoma), cerebrovascular disease and epilepsy. The patients were asked if a medical disease was present or absent. Being diagnosed and treated at any time for the diseases in question was accepted as an inclusion criterion. Patients with alcohol and substance abuse (except nicotine addiction) were excluded from the study. The rate of nicotine addiction was found to be $58.6 \%$ among the patients. Clinical features were recorded as psychotic findings, mixed, atypical, melancholic and catatonic features, seasonal course and comorbid anxiety disorder.

Information regarding the medical histories of the patients' relatives was obtained from the patients. The patients were asked if a physical illness was present or absent, and if present, in whom (degree of consanguinity (in first- or second-degree relatives separately). Being diagnosed and treated at any time for the medical diseases in question was accepted as an inclusion criterion. Relatives with alcohol and substance abuse (except nicotine addiction) were excluded from the study. The rate of nicotine addiction was found to be $31.2 \%$ among patients' relatives. Patient relatives who had died due to one of the conditions above were marked as positive.

The statistical analyses were performed using the SPSS 20.0 software. $\mathrm{P}<0.05$ was considered significant, and all tests were two-tailed.

The Institutional Review Board of Uskudar University approved the study. Patients gave written informed consent in accordance with the Declaration of Helsinki.

\section{Results}

\section{Sample}

The mean age of the sample (612 female, 536 male) was 28.4 \pm 6.2 . The mean age was found to be similar in both sexes (P $=0.323$ ); and $56.4 \%$ of the patients were married and $78.3 \%$ were employed.

The mean age of disorder onset was $21.2 \pm 3.8$. Psychotic features were present in $16.7 \%(\mathrm{n}=192)$, mixed features in $66.6 \%(n=764)$ and seasonal course in $46.7 \%(n=536)$ of the cases.

The rate of diabetes was $28.2 \%(n=324)$, ischemic heart disease $9 \%(n=104)$, thyroid disease $22.9 \%(n=264)$, cancer (gastrointestinal cancers, breast and prostate cancer, leukemia, and lymphoma) $8.7 \%(\mathrm{n}=100)$, cerebrovascular disease $5.6 \%$ $(n=64)$ and epilepsy $3.8 \%(n=44)$ in the sample (Table 1$)$.

\section{Rate of family history of medical disease}

In the study sample, presence of family history for either diabetes, ischemic heart disease, thyroid disease, cancer (gastrointestinal cancers, breast and prostate cancer, leukemia, and lymphoma), cerebrovascular disease or epilepsy was calculated as $92 \%(n=1,056)$. The ratio of the solitary existence rate of any of these diagnoses to their coexistence with any other is defined as specific comorbidity, and the remaining percentage is defined as cumulative comorbidity. The former is related to specificity and the latter with sensitivity.

There was no statistically significant difference regarding the presence of the medical disease in the maternal or the paternal side or first- and second-degree relatives.

Diabetes, ischemic heart disease, cancer (gastrointestinal cancers, breast and prostate cancer, leukemia, and lymphoma), cerebrovascular disease and epilepsy are more commonly seen in the family members than in patients with bipolar affective disorder (Table 1).

\section{Association between family history and clinical properties}

A strong correlation was found between a positive family his- 
tory for epilepsy and mood disorder with psychotic features ( $\mathrm{r}$ $=0.621, \mathrm{P}=0.001)$. A correlation was found between a positive family history of diabetes and seasonal course and also between a positive family history of thyroid disease and comorbid anxiety disorder $(\mathrm{r}=0.307, \mathrm{P}=0.038 ; \mathrm{r}=0.250, \mathrm{P}=0.042)$.

\section{Discussion}

This study is the first in investigating into the prevalence of positive family history of medical disease in bipolar affective disorder, and it revealed many important findings. Diabetes, ischemic heart disease, cancer (breast, prostate, gastrointestinal system cancers (carcinoid and proliferative tumors), leukemia, and lymphoma), cerebrovascular disease and epilepsy were found to be more common in the family history than in bipolar patients.

For bipolar patients, the risk of occurrence of bipolar affective disorder in a first-degree relative is roughly 10 times the risk in a random individual. Risk of the disorder in an identical twin is about $63 \%$. Calculated heritability for bipolar affective disorder in twin studies is about 0.78. Modeling of disease transmission through family studies has suggested that bipolar disorder is most likely caused by at least three interacting susceptibility genes [9].

Contrary to our hypothesis, thyroid disease is found to be more frequent in bipolar cases than in their families. This subgroup which can be defined as family history positive (FH+) thyroid disease (comorbid anxiety disorder group), is a subgroup that can be revealed by linkage data in literature and it corresponds to comorbid panic disorder in which familial aggregation had been shown.

Thus, use of alternative methods of phenotypic definition may help define genetically more homogeneous groups of bipolar subjects. Familial aggregation and linkage data to support the utility of subtyping by the presence of psychotic symptoms, comorbid panic disorder and bipolar type II in families exist for bipolar affective disorder [9].

When the percentage of subjects with a positive family history of physical disease, especially the cumulative comorbidity rate is considered, two groups can be distinguished: diabetes $63.4 \%$, ischemic heart disease $61.3 \%$, proliferative cancers (breast, prostate and gastrointestinal cancers) $46.3 \%$ (cumulative comorbidity: ischemic heart disease $81.8 \%$, diabetes $97.8 \%$, proliferative cancers $99.9 \%$ ) and cerebrovascular disease $29.2 \%$, epilepsy $10.8 \%$, leukemia and lymphoma $7.3 \%$ (cumulative comorbidity: leukemia and lymphoma $52.4 \%$, cerebrovascular disease $48.8 \%$, epilepsy $35.5 \%$ ). Table 1 compares the rate of physical illness between bipolar subjects and family members and in the second group the difference is statistically significant. According to the findings of this study, there is a strong correlation between psychotic features and the presence of epilepsy in the family history. Thus a subgroup of patients with psychotic findings, with a FH+ for epilepsy is differentiated, which is consistent with the subtype with psychotic features described in literature.

A strong correlation was found between diabetes and seasonal course in the first group which had the highest rates of comorbidity. Diabetes is a component of metabolic syndrome of which seasonality is a predictor [10]. The interface between ischemic heart disease, proliferative cancer (breast, prostate and gastrointestinal cancers), metabolic syndrome and bipolar affective disorder involves glucocorticoid/insulin signaling mechanisms and inflammatory effector systems $[5,11,12]$. In the literature, this group matches FH+ bipolar type II. Metabolic syndrome is more common in bipolar affective disorder type II than in bipolar affective disorder type I on epigenetic grounds where seasonality plays a mediating role [6].

Anticipation or worsening severity of illness in successive generations has been observed, suggesting the trinucleotide repeat hypothesis, though subsequent clinical studies have not consistently confirmed this initial clinical observation [6]. In this study, no difference in FH+ physical disease is found between first- and second-degree relatives. Also, we found no difference between maternal and paternal relatives in terms of $\mathrm{FH}+$ physical disease. An excess of clinically defined maternal transmission of bipolar affective disorder had also been observed, indicating mitochondrial inheritance or genomic imprinting, though again, subsequent clinical studies have not consistently replicated the initial observation [1].

Finally, the biological variables or temperamental vulnerabilities as endophenotypes have been found to be familial in individual pedigrees. Whether metabolic syndrome or electroencephalographic abnormality are endophenotypes should be evaluated in future studies. As a matter of fact, the comorbid physical diseases that were studied, except thyroid disease, are also seen in patients' relatives, including non-bipolar relatives, with a ratio similar to the patients', which is more frequent than the general population.

Heredity as a sole factor is far from being able to explain the association between bipolar affective disorder and physical disease. Gene-environment interaction is the precursor of inflammation in bipolar affective disorder. For example, maternal infections during pregnancy increase the risk of bipolar affective disorder as well as schizophrenia in neonates. Accordingly an association was found with influenza neuroligin, genital type herpes simplex virus (HSV) dysbindin, respiratory type COMT and toxoplasma DISC-1 genes [9]. The interaction between individual immunogenetic properties and environmental factors regulates neurodevelopmental sensitivity and endurance in the early stages of life and defines the individual's future clinical profile. Immunogenetic diversity also determines the type and severity of the environmental factor we are vulnerable to, regulates the inflammatory response and governs disease severity and comorbidity.

Toll-like receptors (TLR) are related to molecular patterns that promote pathogenesis. They are responsible for the production of cytokines and chemokines, referred to as innate immunity. They are located in the microglia, thyroid gland, adrenal gland, intestines and for a certain developmental period in the thymus gland [13]. At this point, it is important to remember that the first term designating mood stabilizing agents was thymoleptics. A deficiency in innate immunity results in chronic inflammation. On the other side of the coin is human leukocyte antigen (HLA), as adaptive, in other words acquired immunity. Here the referred adaptation is immunosuppression. At this point, the concepts of autoimmune psychosis and autoimmune mood disorder come to the fore [14]. Encephalitis 
due to antibodies formed against the receptors in the brain, the most typical of which is N-methyl-d-aspartate receptor (NMDA-R) encephalitis, is associated with a predisposition of HLA genes [15].

\section{Conclusions}

Acute and chronic stress responses are mediated by glucocorticoid/insulin signaling mechanisms. In the pathogenesis of multiple etiologies, current treatment strategies target common leading pathways and symptoms. However, interactions between stress and neural circuits underlying the pathophysiology of the disorder should be determined as treatment targets. One size does not fit all. Anti-inflammatory and anti-cytokine drugs are shown to be effective in affective and non-affective psychoses [16]. In two resistant cases bone marrow transplantation eliminated psychotic symptoms [17].

There is a two-way interaction between mood disorders and physical illness. This bidirectional interaction should contribute to the use of biological indicators as our diagnostic tools, help identify individual treatment options based on family history, and develop preventive strategies.

\section{Acknowledgments}

We are grateful to our patients and our institution.

\section{Financial Disclosure}

This research did not receive any specific grant from funding agencies in the public, commercial, or not-for-profit sectors.

\section{Conflict of Interest}

The authors declare no conflict of interest.

\section{Informed Consent}

Patients gave written informed consent for publication.

\section{Author Contributions}

Sermin Kesebir conceived and designed the experiments, performed the experiments, analyzed and interpreted the data with Merve Iris Koc and Ahmet Yosmaoglu. Sermin Kesebir wrote the paper.

\section{Data Availability}

The data supporting the findings of this study are available from the corresponding author upon reasonable request.

\section{References}

1. Kesebir S, Vahip S, Akdeniz F, Yuncu Z, Alkan M, Akiskal H. Affective temperaments as measured by TEMPSA in patients with bipolar I disorder and their first-degree relatives: a controlled study. J Affect Disord. 2005;85(12):127-133.

2. Kesebir S, Yosmaoglu A. QEEG in affective disorder: about to be a biomarker, endophenotype and predictor of treatment response. Heliyon. 2018;4(8):e00741.

3. Krapelin E. Manic depressive Insanity and Paranoia. J Nerv Ment Dis. 1921;53.

4. Mass E, Jacome-Galarza CE, Blank T, Lazarov T, Durham $\mathrm{BH}$, Ozkaya $\mathrm{N}$, Pastore $\mathrm{A}$, et al. A somatic mutation in erythro-myeloid progenitors causes neurodegenerative disease. Nature. 2017;549(7672):389-393.

5. Kesebir S. Metabolic syndrome and childhood trauma: Also comorbidity and complication in mood disorder. World J Clin Cases. 2014;2(8):332-337.

6. Kesebir S, Erdinc B, Tarhan N. Predictors of metabolic syndrome in first manic episode. Asian J Psychiatr. 2017;25:179-183.

7. Kesebir S. Epigenetics of metabolic syndrome as a mood disorder. J Clin Med Res. 2018;10(6):453-460.

8. Yuncu Z, Kesebir S, Akdeniz F, Vahip S. [Anticipation in bipolar disorder: A comparison between two generations]. Turk Psikiyatri Derg. 2008;19(4):349-357.

9. Goodwin FK, Jamison KR. Manic-Depressive Illness. Second Edition. Oxford University Press. Newyork. 2007.

10. Turan C, Kesebir S, Suner O. Are ICAM, VCAM and Eselectin levels different in first manic episode and subsequent remission? J Affect Disord. 2014;163:76-80.

11. Gencer AG, Kesebir S. Diabetes in first episode mania: relations with clinical and the other endocrinological and metabolic parameters. Bipolar Disord. 2012;14:90.

12. Kesebir S, Bayrak A. Bipolar disorder and cancer. Curr Approach Psychiatry. 2012;4:223-236.

13. Savitz JB, Teague TK, Misaki M, Macaluso M, Wurfel BE, Meyer M, Drevets D, et al. Treatment of bipolar depression with minocycline and/or aspirin: an adaptive, $2 \times 2$ double-blind, randomized, placebo-controlled, phase IIA clinical trial. Transl Psychiatry. 2018;8(1):27.

14. Binks S, Varley J, Lee W, Makuch M, Elliott K, Gelfand JM, Jacob S, et al. Distinct HLA associations of LGI1 and CASPR2-antibody diseases. Brain. 2018;141(8):2263-2271.

15. Mueller SH, Farber A, Pruss H, Melzer N, Golombeck KS, Kumpfel T, Thaler F, et al. Genetic predisposition in anti-LGI1 and anti-NMDA receptor encephalitis. Ann Neurol. 2018;83(4):863-869.

16. Kappelmann N, Lewis G, Dantzer R, Jones PB, Khandaker GM. Antidepressant activity of anti-cytokine treatment: a systematic review and meta-analysis of clinical trials of chronic inflammatory conditions. Mol Psychiatry. 2018;23(2):335-343.

17. Miyaoka T, Wake R, Hashioka S, Hayashida M, Oh-Nishi A, Azis IA, Izuhara M, et al. Remission of psychosis in treatment-resistant schizophrenia following bone marrow transplantation: a case report. Front Psychiatry. 2017;8:174. 